\title{
Projection Postulate and Atomic Quantum Zeno Effect
}

\author{
Almut Beige and Gerhard C. Hegerfeldt \\ Institute for Theoretical Physics [ـ] \\ University of Göttingen \\ Bunsenstr. 9 \\ D 37073 Göttingen, Germany
}

\begin{abstract}
The projection postulate has been used to predict a slow-down of the time evolution of the state of a system under rapidly repeated measurements, and ultimately a freezing of the state. To test this so-called quantum Zeno effect an experiment was performed by Itano et al. (Phys. Rev. A 41, 2295 (1990)) in which an atomic-level measurement was realized by means of a short laser pulse. The relevance of the results has given rise to controversies in the literature. In particular the projection postulate and its applicability in this experiment have been cast into doubt. In this paper we show analytically that for a wide range of parameters such a short laser pulse acts as an effective level measurement to which the usual projection postulate applies with high accuracy. The corrections to the ideal reductions and their accumulation over $n$ pulses are calculated. Our conclusion is that the projection postulate is an excellent pragmatic tool for a quick and simple understanding of the slow-down of time evolution in experiments of this type. However, corrections have to be included, and an actual freezing does not seem possible because of the finite duration of measurements.
\end{abstract}

PACS numbers 03.65.Bz; 42.50.-p; 32.80.-t

\section{Introduction}

The so-called quantum Zeno effect (QZE) [1] is a theoretical prediction for the behavior of a system under rapidly repeated measurements at times $\Delta t$ apart. It is based on usual quantum theory and on the concept of instantaneous measurements together with ensuing state reductions according to the projection postulate of von Neumann and Lüders [2]. The predictions are:

1. Impediment and slow-down of the time development of the system due to repeated measurements.

2. Freezing of the state for $\Delta t \rightarrow 0$, i.e. in the limit of continuous measurements.

The underlying reason for this can be traced to the fact that for short enough times transition probabilities grow quadratically with time. If, in a given time interval $T$, one performs $n$ measurements at times $\Delta t=T / n$ apart, then the probability to find an 
orthonormal state is at most proportional to $n(\Delta t)^{2}=T^{2} / n$, which goes to zero for $n \rightarrow \infty$ or $\Delta t \rightarrow 0$.

Properties 1 and 2 may be taken as a definition of the QZE, as for instance in Refs. [3, [4, 6, 6]. A slightly different definition is used in Ref. [7] where essentially only property 2 is used.

An experiment to test the QZE for atomic systems has been performed by Itano et al. [3], following a suggestion by Cook [0]. In the experiment a large number $N$ of ions was stored in a Penning trap (see Fig. 1 for the relevant level structure, a V configuration; level 2 is (meta-)stable). The time development is given by a so-called $\pi$ pulse [8] of length $T_{\pi}$, tuned to the $1-2$ transition frequency. A very short pulse of a probe laser couples level 1 with an auxiliary third level, and this is regarded as a measurement to which the projection postulate is applied as follows. It is assumed that an atom is either in level 1 or level 2, depending on whether or not it has emitted photons during the probe pulse [9]. At the end of the $\pi$ pulse the final level populations for up to 64 probe pulses ("measurements") during a $\pi$ pulse (see Fig. 2) are determined and found to be in agreement with the quantum Zeno predictions [10].

The interpretation of this experiment, as to whether it does or does not bear on the QZE, has been controversial in the literature. Some have hailed the results as a dramatic verification of the QZE, others argue that they are unrelated to it [4, 5, 11, 12, 13, 14, 15, 16, [1]. In particular the use of the projection postulate and reduction of the wavefunction have been criticized, and the very existence of the QZE has been cast into doubt. It is no exaggeration to say that the QZE has aroused tremendous interest in the literature 18, 19, 20.

It was pointed out in Refs. [4, 5, 14, 15, 16, 17] that these results could be understood without any recourse to the projection postulate or to the QZE. One can simply incorporate the short probe pulses in the dynamics by an appropriate term in the Hamiltonian or in the optical Bloch equations. A numerical solution of these Bloch equations should then yield the experimental result, and indeed they do to good agreement. The projection postulate does not seem to be needed. Is it also incorrect to use it here?

This is one of the main two questions we are going to address in this paper. First we give a justification of the projection postulate as a useful and approximate technical tool in experimental situations of the general setup considered in Ref. [3] for a wide range of parameters. Our motivation differs somewhat from that of Refs. [4, 5, 14, 15, 16, 17], although there is no contradiction, in particular not to Refs. [5, 16]. In Ref. [5] the ensemble density matrix is calculated by adiabatic Bloch equation techniques for a single probe pulse and found to be nearly diagonal. But this contains no information about the outcome for individual atoms and subensembles and does not prove that atoms with or without photon emissions are in $|1\rangle$ and $|2\rangle$, respectively. The same technique is used in Ref. [16] to study the parameter domain of "good" measurements with a resulting diagonal density matrix [21]. We, however, are concerned first with selective measurements, namely with the states of atoms at the end of a probe pulse with or without photon emissions. These states are explicitly determined for the first time and found to be close, but not identical, to $|1\rangle$ and $|2\rangle$ [22]. Thus a probe pulse can indeed be regarded as accomplishing a highly accurate - but not perfect - reduction to and measurement of levels 1 and 2 in experiments of the type of Ref. [3] for a wide range of parameters. As a second question we discuss the cumulative effect of the deviations from ideal measurements - i.e. measurements which can be described by the projection postulate - over $n$ probe pulses 
for the density matrix and determine the resulting level populations at the end of the $\pi$ pulse. We exhibit an explicit $n$ dependence of the corrections to the treatment by the projection postulate. This is also a new result.

For a probe pulse to be an effective measurement some obvious requirements have to be fulfilled. First of all, its duration, $\tau_{\mathrm{p}}$, should be very short compared to the duration $T_{\pi}$ of the $\pi$ pulse,

$$
\tau_{\mathrm{p}} \ll T_{\pi} .
$$

Furthermore, the probe pulse cannot be too short or too weak, because it should produce fluorescence photons from level 1 with high certainty. In addition, at the end of a probe pulse any population in the auxiliary level 3 should decay to level 1 extremely rapidly. This means that $A_{3}^{-1}$, the inverse Einstein coefficient of level 3 , must be tiny compared to the time between two probe pulses,

$$
A_{3}^{-1} \ll \frac{T_{\pi}}{n}-\tau_{\mathrm{p}}
$$

The first two conditions lead to a restriction on two parameters, namely 23

$$
\epsilon_{\mathrm{p}} \equiv \frac{A_{3} \Omega_{2}}{\Omega_{3}^{2}} \ll 1 \quad \text { and } \quad \epsilon_{\mathrm{R}} \equiv \frac{\Omega_{2}}{\Omega_{3}} \ll 1 .
$$

In Sections 4 and 5 we will use the conditions

$$
\epsilon_{\mathrm{p}} \equiv \frac{A_{3} \Omega_{2}}{\Omega_{3}^{2}} \ll 1 \quad \text { and } \quad \epsilon_{\mathrm{d}} \equiv \frac{\Omega_{3}^{2}}{A_{3}^{2}} \ll 1 .
$$

Then the condition $\epsilon_{\mathrm{R}} \ll 1$ is automatically fulfilled since $\epsilon_{\mathrm{R}}=\epsilon_{\mathrm{p}} \epsilon_{\mathrm{d}}^{1 / 2}$. This parameter regime is compatible with the one investigated in Ref. [16]. In the experiment of Itano et al. [3] $\epsilon_{\mathrm{R}}$ is about $6.5 \cdot 10^{-6}, \epsilon_{\mathrm{p}}$ about $4.1 \cdot 10^{-4}$, and $\epsilon_{\mathrm{d}}$ about $2.5 \cdot 10^{-4}$ [24.

In our paper we mainly use the quantum jump approach (quantum trajectories) [26, 27, 28], and because of its inherent simplicity the analysis can be carried out analytically here for a wide range of experimental parameters. This approach is equivalent to the the Monte-Carlo wave function approach [29]. The ensemble of all trajectories satisfies the usual Bloch equations and is often used to obtain numerical solutions of the latter through numerical simulations. The quantum jump approach deals with pure states for single atoms instead of density matrices for ensembles [25], which in the present case means a simplification from nine components to three, and it describes the time development of single atoms between photon emissions by a simple reduced Hamiltonian and by a "jump" to a reset state (in this paper the ground state) at a photon detection [30. It allows an intuitive understanding of the processes involved.

The simple derivation of the quantum jump approach outlined in Section 2 uses the projection postulate and reductions as a technical tool for photon detections (not for atomic measurements!), and it might seem that one uses this postulate for an investigation of itself. However, as explained elsewhere [26] the use of reductions in the derivation of quantum trajectories is not necessary and can be avoided. Moreover, the photon detections happen on a much shorter time scale than all times considered in the experiment, and for such detections the projection postulate has been a reliable tool in the past.

In Section 3 we consider the simple case of a probe pulse with the $\pi$ pulse temporarily switched off. In this example it is very easy to see how the probe pulse acts on a state 
$\alpha_{1}|1\rangle+\alpha_{2}|2\rangle$ of a single atom and that it effectively leaves it either in $|1\rangle$ or in $|2\rangle$, with probability $\left|\alpha_{1}\right|^{2}$ and $\left|\alpha_{2}\right|^{2}$, respectively, depending on whether the atom has emitted photons or not. The small deviations from an ideal measurement with perfect state reduction are determined.

In Section 4 we consider the case of a single probe pulse with the $\pi$ pulse switched on. Complications arise now since the $\pi$ pulse causes a small additional transition between 1 and 2. In Section 5 the case of $n$ probe pulses during a single $\pi$ pulse and the build-up of the corrections are considered. It turns out that one gets an excellent approximation for the level populations if in the results for the idealized case one takes the finite duration of the probe pulse into account by neglecting the action of the $\pi$ pulse during this time (see Fig. 3). This simply means that $T_{\pi} / n$ is replaced by $\Delta T=T_{\pi} / n-\tau_{\mathrm{p}}$. At the end of Section 5 we compare our analytical results with those of a numerical solution of the optical Bloch equations for the parameters of the experiment. The agreement is amazing.

In the last section we discuss our result and their significance for the role of the projection postulate in the so-called quantum Zeno effect. Our main conclusion is that, although the projection postulate is not necessary here, it is a useful tool for a fairly accurate description of the measurements involved. It is useful since it gives a quick intuitive understanding of the physical situation - without having to solve unwieldy Bloch equation. Insofar the projection postulate should not be dismissed out of hand. However, it is only approximately valid, because in practice realistic measurements are never ideal nor instantaneous. Therefore the first part of the Zeno effect - impediment and slow-down of the time development - can legitimately be understood by the projection postulate, but the second part - the freezing of the state - is in our opinion an over-idealization.

\section{The quantum jump approach in quantum optics. Quantum trajectories}

In this section we briefly summarize the quantum jump approach used in the subsequent sections. The reader familiar with it can proceed directly to Eqs. (16) - (19). The idea is to describe the radiating atom between photon detections by a reduced (or effective) time evolution operator giving the time development under the condition that no photon has been detected [26]. After a photon detection one has to reset the atom to the reset state ("jump"), with ensuing reduced time development, and so on. For a driven system with many emissions one then obtains a stochastic path, called a quantum trajectory [28]. The general reset state has been determined in Ref. [27]. For a V system as considered in this paper the reset state after an emission is the ground state. The reduced time development together with the reset state provide a complete stochastic description of the time development of the atom [27]. Starting with this description one can then derive the Bloch equations describing an ensemble of radiating atoms [26, 27]. In fact both approaches, quantum jumps and Bloch equations, are possible and equivalent ways to describe the time evolution of an ensemble of fluorescing atoms, but the former is also easy to apply to the emission behavior of a single atom.

We now indicate how to determine the reduced time development operator for the $\mathrm{V}$ system. To be sure that no photon has been detected in a time interval one may imagine measurements on the radiation field in a rapid succession at times $\Delta t$ apart, at $t_{1}<\cdots<t_{m}=t$ say. If in all these measurements no photons are found the state of the 
atom at time $t$ is, by the von Neumann - Lüders projection postulate [31],

$$
\begin{aligned}
&|\phi(t)\rangle=\left|0_{p h}\right\rangle\left\langle 0_{p h}\left|U\left(t_{m}, t_{m-1}\right)\right| 0_{p h}\right\rangle\left\langle 0_{p h}\right| \ldots \\
& \ldots\left|0_{p h}\right\rangle\left\langle 0_{p h}\left|U\left(t_{1}, t_{0}\right)\right| 0_{p h}\right\rangle|\psi\rangle \\
&=\left|0_{p h}\right\rangle U_{\text {red }}\left(t, t_{0}\right)|\psi\rangle .
\end{aligned}
$$

where $\left|0_{p h}\right\rangle$ is the vacuum state, $\left|0_{p h}\right\rangle|\psi\rangle$ the initial state, $U$ the complete time development operator, and where the second equality serves as a definition of the reduced time evolution operator $U_{\text {red }}\left(t, t_{0}\right)$, which acts on atomic states. The time difference between successive measurements has to be chosen short enough to be able to say that at most one photon has been detected in this interval. On the other hand this time difference has to be longer than the inverse transition frequencies [26]. Under these assumptions one can calculate Eq. (5) by means of perturbation theory. With $U_{\text {red }}\left(t, t_{0}\right)$ one has a simple expression for the probability $P_{0}(t)$ that no photon is detected in the interval $[0, t]$ if the atom is in the state $|\psi\rangle$ at $t=0$,

$$
\begin{aligned}
P_{0}(t) & =\||\phi(t)\rangle \|^{2} \\
& =\| U_{\text {red }}\left(t, t_{0}\right)|\psi\rangle \|^{2} .
\end{aligned}
$$

We now apply this to the $\mathrm{V}$ system depicted in Fig. 1. In this system the upper levels 2 and 3 couple to a common ground level 1, with Einstein coefficients $A_{2}$ and $A_{3}$. Later we will consider level 2 to be stable and put $A_{2}=0$. We assume here that $\omega_{32} \equiv \omega_{3}-\omega_{2}$ is in the optical range, i.e. not too small. For simplicity we consider zero detunings of the driving fields, whose (real) Rabi frequencies are denoted by $\Omega_{2}$ and $\Omega_{3}$, respectively. The Hamiltonian in rotating wave approximation is given by [32]

$$
H=H_{A}^{0}+H_{F}^{0}+\sum_{i=2}^{3} \hbar\left\{\frac{1}{2} \Omega_{i}|i\rangle\langle 1| e^{-i \omega_{i} t}+\text { h.c. }\right\}+\sum_{i=2}^{3} \sum_{\mathbf{k} \lambda} \hbar\left\{g_{i \mathbf{k} \lambda} a_{\mathbf{k} \lambda}|i\rangle\langle 1|+\text { h.c. }\right\}
$$

where

$$
g_{i \mathbf{k} \lambda}=i e \mathbf{D}_{i 1} \cdot \epsilon_{\mathbf{k} \lambda}\left(\omega_{\mathbf{k} \lambda} /\left(2 \epsilon_{0} \hbar V\right)\right)^{1 / 2},
$$

with the transition dipole moment $\mathbf{D}_{i 1}=\langle i|\mathbf{X}| 1\rangle$ and $\epsilon_{\mathbf{k} \lambda}$ the polarization vector. $V$ is the quantization volume, later taken to infinity. Going over to an interaction picture with respect to

$$
H_{0}=H_{A}^{0}+H_{F}^{0}
$$

one has

$$
H_{I}(t)=\sum_{i=2}^{3} \hbar\left\{\frac{1}{2} \Omega_{i}|i\rangle\langle 1|+\text { h.c. }\right\}+\sum_{i=2}^{3} \sum_{\mathbf{k} \lambda} \hbar\left\{g_{i \mathbf{k} \lambda} a_{\mathbf{k} \lambda}|i\rangle\langle 1| e^{i\left(\omega_{i}-\omega_{\mathbf{k} \lambda}\right) t}+\text { h.c. }\right\} \text {. }
$$

By $U_{I}$ we denote the corresponding time development operator. With $\Delta t=t_{i}-t_{i-1}$ in the range given above we can calculate in second order perturbation theory the time evolution under the condition that no photon has been detected. From

$$
\begin{aligned}
& \left\langle 0_{p h}\left|U_{I}\left(t_{i}, t_{i-1}\right)\right| 0_{p h}\right\rangle \\
= & 1_{A}-\frac{i}{\hbar} \int_{t_{i-1}}^{t_{i}} d t^{\prime}\left\langle 0_{p h}\left|H_{I}\left(t^{\prime}\right)\right| 0_{p h}\right\rangle-\frac{1}{\hbar^{2}} \int_{t_{i-1}}^{t_{i}} d t^{\prime} \int_{t_{i-1}}^{t^{\prime}} d t^{\prime \prime}\left\langle 0_{p h}\left|H_{I}\left(t^{\prime}\right) H_{I}\left(t^{\prime \prime}\right)\right| 0_{p h}\right\rangle
\end{aligned}
$$


one obtains for the first-order contribution

$$
-i \sum_{i=2}^{3}\left\{\frac{1}{2} \Omega_{i}|i\rangle\langle 1|+\text { h.c. }\right\} \Delta t
$$

and for the second order, omitting terms proportional to $(\Delta t)^{2}$,

$$
\begin{aligned}
& -\sum_{i, j=2}^{3} \sum_{\mathbf{k} \lambda} \int_{t_{i-1}}^{t_{i}} d t^{\prime} \int_{t_{i-1}}^{t^{\prime}} d t^{\prime \prime} g_{i \mathbf{k} \lambda} g_{j \mathbf{k} \lambda}^{*} e^{i\left(\omega_{i}-\omega_{\mathbf{k} \lambda}\right) t^{\prime}} e^{-i\left(\omega_{j}-\omega_{\mathbf{k} \lambda}\right) t^{\prime \prime}}|i\rangle\langle j| \\
= & -\sum_{i, j=2}^{3} \sum_{\mathbf{k} \lambda} \int_{t_{i-1}}^{t_{i}} d t^{\prime} e^{i\left(\omega_{i}-\omega_{j}\right) t^{\prime}} \int_{0}^{t^{\prime}-t_{i-1}} d \tau g_{i \mathbf{k} \lambda} g_{j \mathbf{k} \lambda}^{*} e^{i\left(\omega_{j}-\omega_{\mathbf{k} \lambda}\right) \tau}|i\rangle\langle j| .
\end{aligned}
$$

In the last equation we have substituted $\tau=t^{\prime}-t^{\prime \prime}$. Since $\Delta t$ is much larger than the inverse optical frequencies $\omega_{i}^{-1}$ one can extend the inner integral to infinity, leading to $\pi \delta\left(\omega_{j}-\omega_{\mathbf{k} \lambda}\right)$ plus a principle value. Alternatively one can argue that the correlation function

$$
\kappa_{i j}(\tau)=\sum_{\mathbf{k} \lambda} g_{i \mathbf{k} \lambda} g_{j \mathbf{k} \lambda}^{*} e^{i\left(\omega_{j}-\omega_{\mathbf{k} \lambda}\right) \tau}
$$

is negligible for $\tau \gg \omega_{j}^{-1}$. The sum over $\mathbf{k}$ then yields generalized decay constant $\Gamma_{i j}$ and level shifts $\Delta_{i j}$. The level shifts are small [33] and will be neglected in the following. With

$$
\Gamma_{i j}=\frac{e^{2} \mathbf{D}_{i 1} \cdot \mathbf{D}_{1 j}}{6 \pi \epsilon_{0} \hbar c^{3}} \omega_{j}^{3}
$$

one obtains for the second-order contribution

$$
-\sum_{i, j=2}^{3} \Gamma_{i j}|i\rangle\langle j| \int_{0}^{\Delta t} d \tau e^{-i\left(\omega_{i}-\omega_{j}\right) \tau}
$$

Now, if $\omega_{3}-\omega_{2}$ is in the optical range, as supposed here, then the last integral vanishes for $i \neq j$ and equals $\Delta t$ otherwise. We note that

$$
\Gamma_{i i}=\frac{1}{2} A_{i}
$$

where $A_{i}$ is the Einstein coefficient of the $i$-th level. Collecting all terms we thus obtain

$$
\left\langle 0_{p h}\left|U_{I}\left(t_{i}, t_{i-1}\right)\right| 0_{p h}\right\rangle=\mathbf{1}_{A}-i \sum_{i=2}^{3}\left\{\frac{1}{2} \Omega_{i}|i\rangle\langle 1|+\text { h.c. }\right\} \Delta t-\sum_{i=2}^{3} \frac{1}{2} A_{i}|i\rangle\langle i| \Delta t .
$$

This can be written as $\exp \left\{-i H_{\text {red }}^{I} \Delta t / \hbar\right\}$ where, in matrix notation, the reduced Hamiltonian $H_{\text {red }}^{I}$ and the atomic operator $M$ are defined through

$$
H_{\mathrm{red}}^{I} / \hbar=\frac{1}{2}\left(\begin{array}{ccc}
0 & \Omega_{2} & \Omega_{3} \\
\Omega_{2} & -i A_{2} & 0 \\
\Omega_{3} & 0 & -i A_{3}
\end{array}\right) \equiv-i M
$$

Later on, we will take $A_{2}=0$. For arbitrary time intervals we thus have, in the interaction picture,

$$
U_{\text {red }}^{I}(t, 0)=e^{-i H_{\text {red }}^{I} t / \hbar} \equiv e^{-M t}
$$


The no-photon probability is then, for $t_{0}=0$ and initial state $\psi$, or more generally a density matrix $\rho(0)$,

$$
\begin{aligned}
P_{0}(t ; \psi) & =\| e^{-M t}|\psi\rangle \|^{2} \\
P_{0}(t ; \rho(0)) & =\operatorname{tr}\left\{e^{-M t} \rho(0) e^{-M^{\dagger} t}\right\} .
\end{aligned}
$$

The probability that the first photon is emitted in $(t, t+d t)$ equals $P_{0}(t ; \psi)-P_{0}(t+d t ; \psi) \equiv$ $w_{1}(t ; \psi) d t$ where

$$
w_{1}(t ; \psi)=-\frac{d}{d t} P_{0}(t ; \psi)
$$

is the probability density for the first photon [34]. For small upper level separation nonzero off-diagonal $\Gamma_{i j}$ terms may appear which lead to interesting coherence effects [35, 36, 37, 38]. For general $n$-level systems the reduced Hamiltonian is given in Ref. [27].

The reduced time development is not unitary. The reason is that it does not describe the time evolution of the whole ensemble but that of the subensemble with no photons. The size of this subensemble is decreasing in time since an atom for which a photon has been detected leaves the sub-ensemble, and this is reflected by the decrease of the norm squared in Eq. (7). The above probability density determines the (random) time for the first photon. After that the atom is reset to the ground state, $|1\rangle$, for a V system. The next emission time is then determined by $w_{1}(t ; 1)$, and so on. In this way one obtains a quantum trajectory.

From this description of single systems one can recover the usual Bloch equations of the complete ensemble as follows [27]. The density matrix $\rho(t)$ of the ensemble is a sum of two terms, $\rho^{>}$and $\rho^{0}$, corresponding to a subensemble of atoms with or without photon emissions until time $t$, respectively. From Eq. (17) one has

$$
\rho^{0}(t ; \rho(0))=e^{-M t} \rho(0) e^{-M^{\dagger} t} .
$$

If $I(\tau ; \rho(0)) d \tau$ denotes the (unconditioned) probability to find a photon between $\tau$ and $\tau+d \tau$, then the sub-subensemble of atoms with their last emission before $t$ in this interval is described by

$$
I(\tau ; \rho(0)) d \tau \rho^{0}(t-\tau ;|1\rangle)
$$

and therefore

$$
\rho^{>}(t)=\int_{0}^{t} d \tau I(\tau ; \rho(0)) \rho^{0}(t-\tau ;|1\rangle) .
$$

Differentiation of $\rho=\rho^{0}+\rho^{>}$gives

$$
\dot{\rho}(t)=\dot{\rho}^{0}(t ; \rho(0))+I(t ; \rho(0))|1\rangle\langle 1|+\int_{0}^{t} d \tau I(\tau ; \rho(0)) \dot{\rho}^{0}(t-\tau ;|1\rangle) .
$$

Taking the trace and using $\operatorname{tr} \rho(t) \equiv 1$ gives $I(t ; \rho(0))=A_{2} \rho_{22}+A_{3} \rho_{33}$. From Eq. (20) one obtains $\dot{\rho}^{0}$, and inserting this into Eq. (23) gives

$$
\dot{\rho}=-\frac{i}{\hbar}\left[H_{\text {red }}^{I} \rho-\rho H_{\text {red }}^{I \dagger}\right]+\left(A_{2} \rho_{22}+A_{3} \rho_{33}\right)|1\rangle\langle 1| .
$$

This is a compact form of the Bloch equations used in Refs. 四 司. 
In this outline of the quantum jump approach state reductions were used as a tool. But it is noteworthy that one can also use the Markov approximation, indicating a close connection between the two [26].

\section{A simple special case: Intermittent probe and $\pi$ pulse.}

The quantum jump approach will now be applied to the experimental situation of Itano et al. [3]. Here one can take $A_{2}=0$. The simplicity of the mechanism becomes particularly clear if the $\pi$ pulse is switched off while the probe pulse is on. Then $\Omega_{2}$ is zero during a probe pulse and Eq. (16) reads during this time interval

$$
H_{\text {red }}^{I} / \hbar=\frac{1}{2}\left(\begin{array}{ccc}
0 & 0 & \Omega_{3} \\
0 & 0 & 0 \\
\Omega_{3} & 0 & -i A_{3}
\end{array}\right) \equiv-i M_{0} .
$$

Note that this annihilates the state $|2\rangle$ and therefore the reduced time development leaves $|2\rangle$ invariant. At the end of a probe pulse one has to wait a short transient time of the order of $A_{3}^{-1}$ for a possible 3 -component to decay. This will always be done in the following.

(i) Effective reduction by a probe pulse

If the state at the beginning of a probe pulse is

$$
|\psi\rangle=\alpha_{1}|1\rangle+\alpha_{2}|2\rangle
$$

and $0 \leq \tau \leq \tau_{\mathrm{p}}$, then $|\psi\rangle$ evolves, until the emission of the first photon, as

$$
e^{-i H_{\text {red }}^{I} \tau / \hbar}|\psi\rangle=e^{-M_{0} \tau}|\psi\rangle=\alpha_{1} e^{-M_{0} \tau}|1\rangle+\alpha_{2}|2\rangle
$$

since the $\pi$ pulse is assumed to be switched off now. Due to the term $A_{3}$ in $M_{0}$ the norm of the first term of the right hand side decreases exponentially and the first term becomes negligible for $\tau$ large enough. Therefore, if an atom did not emit a photon until the end of the probe pulse it will essentially be in the state $|2\rangle$, and this happens with probability given by the norm-squared of the r.h.s., i.e. by $\left|\alpha_{2}\right|^{2}$ for large enough $\tau_{\mathrm{p}}$.

On the other hand, if an atom does emit one or more photons - this happens with probability $1-\| e^{-M_{0} \tau}|\psi\rangle \|^{2}=\left|\alpha_{1}\right|^{2}\left(1-\|\left. e^{-M_{0} \tau}|1\rangle\right|^{2}\right)$ - then right thereafter it is in state $|1\rangle$ and will then be pumped between $|1\rangle$ and $|3\rangle$ by the probe pulse, with photon emissions. A short time after the end of the probe pulse, $|3\rangle$ decays to $|1\rangle$ due to the damping term $A_{3}$. Thus a single atom is projected onto $|1\rangle$ or $|2\rangle$ by the probe pulse with probability $\left|\alpha_{1}\right|^{2}$ and $\left|\alpha_{2}\right|^{2}$ if $\| e^{-M_{0} \tau}|1\rangle \|^{2}$ can be neglected. For an ensemble of atoms the density matrix becomes diagonal because of the reduction of every single atomic state.

The preceding analysis is easily made more quantitative as follows. The eigenvalues of $M_{0}$ are $\lambda_{2}=0$ and

$$
\lambda_{1,3}=\frac{1}{4}\left(A_{3} \pm \sqrt{A_{3}^{2}-4 \Omega_{3}^{2}}\right) .
$$

The first term on the r.h.s. of Eq. (27) becomes

$$
\alpha_{1} e^{-M_{0} \tau}|1\rangle=\alpha_{1} \frac{1}{\lambda_{1}-\lambda_{3}}\left\{\left(M_{0}-\lambda_{3}\right) e^{-\lambda_{1} \tau}-\left(M_{0}-\lambda_{1}\right) e^{-\lambda_{3} \tau}\right\}|1\rangle,
$$


as immediately checked by explicit differentiation [39]. For $2 \Omega_{3} \leq A_{3}$ the root in Eq. (28) is real and $\lambda_{1}$ and $\lambda_{3}$ are positive. Therefore in this case the exponential decrease goes at least as

$$
\exp \left[-\frac{\tau}{4}\left(A_{3}-\sqrt{A_{3}^{2}-4 \Omega_{3}^{2}}\right)\right] \leq \exp \left[-\frac{\tau}{2} \Omega_{3}^{2} / A_{3}\right]
$$

and this becomes exponentially small for a probe pulse of length $\tau_{\mathrm{p}}$ with

$$
\tau_{\mathrm{p}} \gg 2 A_{3} / \Omega_{3}^{2} \quad\left(\text { for } 2 \Omega_{3}<A_{3}\right) \text {. }
$$

If $4 \Omega_{3}^{2} \geq A_{3}^{2}$ the root is imaginary and the decrease goes as

$$
\exp \left[-\frac{\tau}{4} A_{3}\right]
$$

and Eq. (31) is replaced by

$$
\tau_{\mathrm{p}} \gg 4 / A_{3} \quad\left(\text { for } 2 \Omega_{3}>A_{3}\right) .
$$

This can be combined to

$$
\tau_{\mathrm{p}} \gg \max \left\{A_{3}^{-1}, A_{3} / \Omega_{3}^{2}\right\} .
$$

For the special case under consideration this is the condition on the length of the probe pulse for an effective reduction to $|1\rangle$ and to $|2\rangle$, with probability $\left|\alpha_{1}\right|^{2}$ and $\left|\alpha_{2}\right|^{2}$, respectively.

\section{(ii) Population vs. observed photons}

A single atom is projected onto the ground state if it emits several photons during the probe pulse. For an ensemble of atoms the number of photons is expected to be a measure for the population of level 1 . With the quantum jump approach this is easily seen as follows.

The probability for no photon emission until time $\tau, P_{0}(\tau ; \psi)$, is given by the norm squared of the r.h.s. of Eq. (27), according to Eq. (18), and it approaches $\left|\alpha_{1}\right|^{2}$ for large times. For the subensemble of atoms with emissions the (conditional) probability density for the emission of the first photon is therefore

$$
w_{1}(\tau ; \psi) /\left|\alpha_{1}\right|^{2}=-\frac{d}{d \tau} P_{0}(\tau ; \psi) /\left|\alpha_{1}\right|^{2}
$$

for $\alpha_{1} \neq 0$ (for $\alpha_{1}=0$ it is not defined). Since the two terms on the r.h.s. of Eq. (27) are orthogonal, the $\alpha_{2}$ term drops out upon differentiation, and $-d / d \tau P_{0}(\tau ; \psi)$ is proportional to $\left|\alpha_{1}\right|^{2}$. Thus for $\alpha_{1} \neq 0$ the probability density for the first photon as well as the number of photons per atom in this subensemble (i.e. the conditional expectation value) is independent of the atomic state at the beginning of the probe pulse. The number, $N(\tau ; \psi)$, of photons per atom for an unconditioned ensemble with initial state $|\psi\rangle$ (i.e. the usual expectation value) is

$$
N(\tau ; \psi)=\left|\alpha_{1}\right|^{2} N(\tau ; 1) .
$$

This expression is now also true for $\alpha_{1}=0$. 
The result is exact for the case $\Omega_{2}=0$ and for all times $\tau$ within the validity domain of the quantum jump approach and Bloch equations. As a consequence, in the case of an ensemble realized by a large number of noninteracting atoms without cooperative effects, as in Ref. [3], the number of observed photons per atom is proportional to the population of level 1 .

\section{(iii) Effectiveness of state reduction}

Instead of the condition in Eq. (34) for $\tau_{\mathrm{p}}$ one can use $N\left(\tau_{\mathrm{p}} ; \psi\right)$, the number of photons per atom emitted until time $\tau_{\mathrm{p}}$, as a more precise measure for the effectiveness of state reduction. Eq. (34) corresponds to $N\left(\tau_{\mathrm{p}} ; 1\right) \gg 1$, but we will show that also for smaller $N\left(\tau_{\mathrm{p}} ; \psi\right)$ an almost complete state reduction is obtained.

As pointed out in (i) above, atoms with photon emissions are in $|1\rangle$ if one waits at the end of the probe pulse for a short transient time to allow for the decay of level $|3\rangle$. But atoms without emissions, however, still contain a part which is not reduced to $|2\rangle$ if $\alpha_{1} \neq 0$ in Eq. (27). At the end of the probe pulse and after a short transient time to allow for the decay of level $|3\rangle$ this non-reduced component is

$$
\alpha_{1}\left\langle 1\left|e^{-M_{0} \tau_{\mathrm{P}}}\right| 1\right\rangle|1\rangle .
$$

The smaller the norm of this, the better the reduction to $|2\rangle$. The norm can be estimated by

$$
\| \alpha_{1}\left\langle 1\left|e^{-M_{0} \tau_{\mathrm{p}}}\right| 1\right\rangle|1\rangle\|\leq\| \alpha_{1} e^{-M_{0} \tau_{\mathrm{p}}}|1\rangle \| .
$$

For initial state $|1\rangle$ the number of photons per atom until time $\tau_{\mathrm{p}}, N\left(\tau_{\mathrm{p}} ; 1\right)$, is in good approximation given by the steady state emission rate [32] multiplied by $\tau_{\mathrm{p}}$,

$$
N\left(\tau_{\mathrm{p}} ; 1\right)=A_{3} \frac{\Omega_{3}^{2}}{A_{3}^{2}+2 \Omega_{3}^{2}} \tau_{\mathrm{p}} .
$$

For initial state $|\psi\rangle=\alpha_{1}|1\rangle+\alpha_{2}|2\rangle$ we denote the number of photons per atom until time $\tau_{\mathrm{p}}$ by $N$, i.e. $N=N\left(\tau_{\mathrm{p}}, \psi\right)$. Then one can use Eqs. (36) and (39) to express $\tau_{\mathrm{p}}$ through $N$ and $\left|\alpha_{1}\right|^{2}$,

$$
\tau_{\mathrm{p}}=\frac{A_{3}^{2}+2 \Omega_{3}^{2}}{A_{3} \Omega_{3}^{2}} N /\left|\alpha_{1}\right|^{2} .
$$

This can now be inserted into Eq. (38) to obtain an estimate of the non-reduced part when $\mathrm{N}$ photons per atom are emitted. This norm is easily calculated by Eq. (27). The norm is a function of $\Omega_{3} / A_{3}, N$ and $\alpha_{1}$, and one easily shows that for fixed other parameters it becomes largest for $\left|\alpha_{1}\right|=1$. A graphical evaluation gives as upper bound for the norm of the non-reduced part

$$
\| \alpha_{1}\left\langle 1\left|e^{-M_{0} \tau_{\mathrm{P}}}\right| 1\right\rangle|1\rangle \| \leq 1.04 \cdot e^{-N(\tau ; 1) / 2}
$$

which holds for $N \geq 2$ and for all values of $\Omega_{3}, A_{3}$, and $\alpha_{1}$. For increasing $N$ the reduction thus becomes very effective.

For particular values of $\Omega_{3} / A_{3}$ the bound for the non-reduced part can be substantially improved. E.g., for $\Omega_{3}$ in the vicinity of $A_{3} / 2$ the non-reduced part becomes much smaller than the above bound. For very small and very large values of $\Omega_{3} / A_{3}$ the reduction is somewhat less efficient than for $\Omega_{3}$ close to $A_{3} / 2$. 


\begin{tabular}{|c|c|c|c|c|c|c|c|}
\hline $\mathrm{N}$ & 4 & 5 & 6 & 8 & 10 & 20 & 50 \\
\hline$\Omega_{3} \ll A_{3}$ & 0.135 & 0.082 & 0.050 & 0.018 & $6.7 \cdot 10^{-3}$ & $4.5 \cdot 10^{-5}$ & $1.4 \cdot 10^{-11}$ \\
\hline$\Omega_{3}=A_{3} / 2$ & 0.023 & 0.006 & 0.002 & 0.0001 & $6.7 \cdot 10^{-6}$ & $4.0 \cdot 10^{-12}$ & $2.9 \cdot 10^{-31}$ \\
\hline$\Omega_{3}=A_{3}$ & 0.051 & 0.027 & 0.015 & 0.004 & $6.9 \cdot 10^{-4}$ & $4.3 \cdot 10^{-7}$ & $5.1 \cdot 10^{-17}$ \\
\hline$\Omega_{3}=2 A_{3}$ & 0.094 & 0.065 & 0.038 & 0.011 & $3.4 \cdot 10^{-3}$ & $1.2 \cdot 10^{-5}$ & $5.7 \cdot 10^{-13}$ \\
\hline
\end{tabular}

Table 1: Maximally possible non-reduced part for given number $N$ of observed photons per atom and for different values of $\Omega_{3} / A_{3}$. The $\pi$ pulse switched off.

In Table 1 we have listed the norm of the maximally possible non-reduced part for various values of $N$ and $\Omega_{3} / A_{3}$. The best reduction occurs for $\Omega_{3}$ around $A_{3} / 2$, but the reduction is also excellent for small and large values of $\Omega_{3}$ if $N$ is larger than 8 .

Summarizing this section, we have shown the following for the case in which the $\pi$ pulse is turned off during a probe pulse.

- The probe pulse provides an effective reduction of the initial state provided its duration is much longer than $\max \left\{1 / A_{3}, A_{3} / \Omega_{3}^{2}\right\}$, a rather mild restriction.

- For an (infinite) ensemble the observed number of photons per atom is proportional to the population of level 1.

- Already for small average numbers of emitted photons an almost complete state reduction is obtained.

\section{Simultaneous probe and $\pi$ pulse}

Now we consider a single probe pulse with the $\pi$ pulse switched on. At the end of a probe pulse, we include a short transient time of the order $A_{3}^{-1}$ to allow for the decay of the auxiliary level 3. Since this short transient time, with the action of the $\pi$ pulse, is neglected this introduces an error of the order $\Omega_{2} / A_{3}$ in the time development of the above subensembles. In the following we will assume that this error is much smaller than $\epsilon_{\mathrm{p}}$. Since $\epsilon_{\mathrm{p}}=A_{3} \Omega_{2} / \Omega_{3}^{2}$ this is equivalent to the condition

$$
\epsilon_{\mathrm{d}} \equiv \Omega_{3}^{2} / A_{3}^{2} \ll 1
$$

In this and the next section we will use the conditions $\epsilon_{\mathrm{p}}, \epsilon_{\mathrm{d}} \ll 1$. The condition $\epsilon_{\mathrm{R}} \ll 1$ is then automatically fulfilled [24.

The $\pi$ pulse causes a small additional transition between 1 and 2 . We will show that, as a consequence, an atom with initial state $|\psi\rangle=\alpha_{1}|1\rangle+\alpha_{2}|2\rangle$ and without photon emission until the end of a probe pulse, including the above short transient time, is not in the state $|2\rangle$ but in a state $|\tilde{\lambda}\rangle$ which also has a 1-component. On the other hand, if an atom emits photons, the last photon may have been emitted some time before the end of the probe pulse. Right after the emission the atom is in $|1\rangle$, but until the end of the probe pulse a small contribution of state $|2\rangle$ may build up, due to the action of the 
$\pi$ pulse. Thus the atom will not be in $|1\rangle$ as in the ideal projection result. Instead it is in a mixed state, denoted by $\tilde{\rho}$. Thus, with the $\pi$ pulse switched on, a single probe pulse effectively projects onto the state $|\tilde{\lambda}\rangle$ if no photon is emitted and onto $\tilde{\rho}$ otherwise, and this happens with the probability $P_{0}\left(\tau_{\mathrm{p}} ; \psi\right)$ and $1-P_{0}\left(\tau_{\mathrm{p}} ; \psi\right)$, respectively. In the following $|\tilde{\lambda}\rangle$ and $\tilde{\rho}$ will be determined. If $\epsilon_{\mathrm{p}}, \epsilon_{\mathrm{d}} \ll 1$ the differences between $|\tilde{\lambda}\rangle$ and $|2\rangle$, $\tilde{\rho}$ and $|1\rangle\langle 1|$ and $P_{0}\left(\tau_{\mathrm{p}} ; \psi\right)$ and $\left|\alpha_{2}\right|^{2}$ are small and the results of Section 3 can be used as a good approximation.

A single atom now evolves with the reduced Hamiltonian $H_{\text {red }}^{I} \equiv-i \hbar M$ of Eq. (16), with $A_{2}=0$, until the emission of the first photon. The possible pumping between levels 1 and 2 is reflected by the fact that $|2\rangle$ is no longer annihilated by $H_{\text {red }}^{I}$. To calculate the time development $\exp \left\{-i H_{\text {red }}^{I} t / \hbar\right\}=\exp \{-M t\}$ one may proceed in two alternative ways if the eigenvalues $\lambda_{i}$ of $M, i=1,2,3$, are all distinct. In the first way one determines the corresponding eigenvectors $\left|\lambda_{i}\right\rangle$ of $M$. Since $M$ is non-hermitian these are in general nonorthogonal, and therefore one also needs the reciprocal basis $\left\{\left|\lambda^{i}\right\rangle\right\}$ with $\left\langle\lambda^{i} \mid \lambda_{j}\right\rangle=\delta_{i j}$. Then one can write

$$
e^{-M t}=\sum e^{-\lambda_{i} t}\left|\lambda_{i}\right\rangle\left\langle\lambda^{i}\right|
$$

Alternatively one has, as a generalization of Eq. (29),

$$
e^{-M t}=e^{-\lambda_{1} t} \frac{\left(M-\lambda_{2}\right)\left(M-\lambda_{3}\right)}{\left(\lambda_{1}-\lambda_{2}\right)\left(\lambda_{1}-\lambda_{3}\right)}+\text { cyclic permutations }
$$

which is immediately checked by application to eigenvectors. The case of degenerate eigenvalues can be treated by considering appropriate limits of Eq. (44).

Comparing the two equations one sees that $\left|\lambda_{i}\right\rangle\left\langle\lambda^{i}\right|$ coincides with the operator multiplying $e^{-\lambda_{i} t}$ in Eq. (44). Moreover, applying this operator to any vector gives a multiple of $\left|\lambda_{i}\right\rangle$, thus automatically yielding the eigenvectors. The eigenvalues are the roots of the characteristic equation [41] which, in principle, can be solved in closed form. One easily calculates

$$
\left(M-\lambda_{1}\right)\left(M-\lambda_{3}\right)\left(\begin{array}{l}
1 \\
0 \\
0
\end{array}\right)=\left(\begin{array}{c}
\lambda_{2}\left(\lambda_{2}-\frac{1}{2} A_{3}\right) \\
\frac{1}{2} i \Omega_{2}\left(\lambda_{2}-\frac{1}{2} A_{3}\right) \\
\frac{1}{2} i \lambda_{2} \Omega_{3}
\end{array}\right) .
$$

By the above remarks, this is a multiple of $\left|\lambda_{2}\right\rangle$. Similarly 42,

$$
\left(M-\lambda_{1}\right)\left(M-\lambda_{3}\right)\left(\begin{array}{l}
0 \\
1 \\
0
\end{array}\right)=-\frac{\Omega_{2}}{2 i \lambda_{2}}\left(M-\lambda_{1}\right)\left(M-\lambda_{3}\right)\left(\begin{array}{l}
1 \\
0 \\
0
\end{array}\right),
$$

which is also a multiple of $\left|\lambda_{2}\right\rangle$.

For the parameter range of the Introduction good approximations for $\lambda_{i}$ are

$$
\begin{aligned}
\lambda_{1,3} & =\frac{1}{4}\left(A_{3} \pm \sqrt{A_{3}^{2}-4 \Omega_{3}^{2}}\right) \\
\lambda_{2} & =\frac{1}{2} A_{3} \Omega_{2}^{2} / \Omega_{3}^{2}=\frac{1}{2} \epsilon_{\mathrm{R}}^{2} A_{3}
\end{aligned}
$$

where $\lambda_{2}$ has been obtained by Newton's method. Note that, for $\Omega_{2} \ll \Omega_{3}$,

$$
\lambda_{2} \ll \operatorname{Re} \lambda_{1,3} \text {. }
$$


Hence the exponentials $\exp \left\{-\lambda_{1,3} t\right\}$ in Eq. (44) drop off very rapidly. When calculating $\exp \left\{-M \tau_{\mathrm{p}}\right\}|\psi\rangle$ by Eq. (44) one can therefore, as in Section 3, neglect the $\left|\lambda_{1}\right\rangle$ and $\left|\lambda_{3}\right\rangle$ terms if $\tau_{\mathrm{p}} \gg \max \left\{A_{3}^{-1}, A_{3} / \Omega_{3}^{2}\right\}=A_{3} / \Omega_{3}^{2}$ (because $\epsilon_{\mathrm{d}} \ll 1$ ).

\section{(i) Subensemble without photon emission}

With Eqs. (44) to (47) one can now obtain the state of the subensemble of atoms without photon emissions until the end [40] of the probe pulse. For initial state $|\psi\rangle$,

$$
|\psi\rangle=\alpha_{1}|1\rangle+\alpha_{2}|2\rangle,
$$

it is described at time $\tau_{\mathrm{p}}$ by

$$
\begin{aligned}
e^{-M \tau_{\mathrm{p}}}|\psi\rangle & =e^{-M \tau_{\mathrm{p}}}\left\{\alpha_{1}|1\rangle+\alpha_{2}|2\rangle\right\} \\
& =e^{-\lambda_{2} \tau_{\mathrm{p}}}\left(\alpha_{2}-i \epsilon_{\mathrm{p}} \alpha_{1}\right) \sqrt{1-\epsilon_{\mathrm{R}}^{2}+\epsilon_{\mathrm{p}}^{2}}\left|\lambda_{2}\right\rangle
\end{aligned}
$$

where

$$
\left|\lambda_{2}\right\rangle=\frac{1}{\sqrt{1-\epsilon_{\mathrm{R}}^{2}+\epsilon_{\mathrm{p}}^{2}}}\left(\begin{array}{c}
-i \epsilon_{\mathrm{p}} \\
1-\epsilon_{\mathrm{R}}^{2} \\
\epsilon_{\mathrm{R}}
\end{array}\right)
$$

Moreover,

$$
\lambda_{2} \tau_{\mathrm{p}}=\frac{1}{2} \epsilon_{\mathrm{p}} \pi \frac{\tau_{\mathrm{p}}}{T} \ll 1
$$

and hence $\exp \left\{-\lambda_{2} \tau_{\mathrm{p}}\right\} \approx 1$. Similarly for an initial density matrix. The state $\left|\lambda_{2}\right\rangle$ in Eq. (49) has a very small third component, and after the probe pulse has been turned off this component will, on the time scale of $A_{3}^{-1}$, decay to zero [43]. Thus at the end of a probe pulse and after this transient decay time the subensemble with no photons is in the normalized - state

$$
|\tilde{\lambda}\rangle \equiv \mathbb{P}_{1,2}\left|\lambda_{2}\right\rangle /\|\cdot\|=\frac{1}{\sqrt{1+\epsilon_{\mathrm{p}}^{2}}}\left(\begin{array}{c}
-i \epsilon_{\mathrm{p}} \\
1 \\
0
\end{array}\right) \approx|2\rangle
$$

where $\mathbb{P}_{1,2} \equiv|1\rangle\langle 1|+| 2\rangle\langle 2|$ denotes the projector onto the 1-2 subspace and where terms

of higher orders in $\epsilon_{\mathrm{p}}$ and $\epsilon_{\mathrm{d}}$ have been omitted. The probability for no photon emission is

$$
\begin{aligned}
P_{0}\left(\tau_{\mathrm{p}} ; \psi\right) & =|| \mathbb{P}_{1,2} e^{-M \tau_{\mathrm{p}}}|\psi\rangle \|^{2} \\
& =\left|\alpha_{2}\right|^{2}+2 \operatorname{Im} \alpha_{1} \alpha_{2}^{*} \epsilon_{\mathrm{p}}-\pi\left|\alpha_{2}\right|^{2} \frac{\tau_{\mathrm{p}}}{T_{\pi}} \epsilon_{\mathrm{p}}+\text { higher orders in } \epsilon_{\mathrm{p}}, \epsilon_{\mathrm{d}}
\end{aligned}
$$

For an initial density matrix $\rho^{\text {in }}$ instead of the pure state $\psi$ one has to replace $\left|\alpha_{2}\right|^{2}$ by $\rho_{22}^{\text {in }}$ and $\alpha_{1} \alpha_{2}^{*}$ by $\rho_{12}^{\text {in }}$.

Thus, to good approximation, the probability for no photon emission is proportional to the population of level 2 and the atoms with no emissions are approximately in the state $|2\rangle$. For the parameters [44] of the experiment [3] the corrections in Eq. (52) are less than $4 \cdot 10^{-4}$. 


\section{(ii) Subensemble with photon emissions}

We will now calculate the density matrix $\tilde{\rho}$ for the subensemble with photon emissions. One can employ a systematic expansion in powers of $\Omega_{2}$, including second order. However, the following more physically motivated procedure is simpler and yields the same results.

At the beginning of the probe pulse we assume the ensemble to be in the pure state $|\psi\rangle$; for a density matrix the treatment is similar. The complete ensemble at time $\tau_{\mathrm{p}}$ after the beginning of the probe pulse can be thought of as consisting of two subensembles of atoms with and without photon emissions. The latter is described by Eq. (20),

$$
\rho^{0}\left(\tau_{\mathrm{p}} ; \psi\right) \equiv e^{-M \tau_{\mathrm{p}}}|\psi\rangle\langle\psi| e^{-M^{\dagger} \tau_{\mathrm{p}}}
$$

with its relative weight given by Eq. (18),

$$
\operatorname{tr} \rho^{0}\left(\tau_{\mathrm{p}} ; \psi\right)=P_{0}\left(\tau_{\mathrm{p}} ; \psi\right)
$$

According to Eq. (22) the former subensemble is described by

$$
\rho^{>}\left(\tau_{\mathrm{p}} ; \psi\right)=\int_{0}^{\tau_{\mathrm{p}}} d \tau I(\tau ; \psi) \rho^{0}\left(\tau_{\mathrm{p}}-\tau ; 1\right)
$$

The complete density matrix is $\rho^{>}+\rho^{0}$, and therefore

$$
\operatorname{tr} \rho^{>}=1-P_{0} \text {. }
$$

We now determine $\rho_{12}^{>}$and $\rho_{22}^{>}$at time $\tau_{\mathrm{p}}$. For initial state $|1\rangle$ the no-photon probability decreases rapidly, and therefore $\rho^{0}\left(\tau_{\mathrm{p}}-\tau ; 1\right)$ contributes essentially only in the vicinity of $\tau \approx \tau_{\mathrm{p}}$ (cf. Eqs. (29) to (31)). Because of this one can replace $I(\tau ; \psi)$ by $I\left(\tau_{\mathrm{p}} ; \psi\right)$ which is approximately equal to the fraction $1-P_{0}\left(\tau_{\mathrm{p}} ; \psi\right)$ of emitting atoms times $I\left(\tau_{\mathrm{p}} ; 1\right)$. The latter practically equals the stationary emission rate for the three-level system which is, up to terms of order in $\Omega_{2}^{2}$, the stationary rate from Eq. (39) for the two-level system. For

the calculation of the 12 and 22 component these corrections in $\Omega_{2}^{2}$ can be omitted since $\rho^{0}\left(\tau_{\mathrm{p}}-\tau ; 1\right)_{12}$ and $\rho^{0}\left(\tau_{\mathrm{p}}-\tau ; 1\right)_{22}$ are themselves of order $\Omega_{2}$ and $\Omega_{2}^{2}$ and much smaller than 1. Thus we obtain

$$
\rho^{>}\left(\tau_{\mathrm{p}} ; \psi\right)_{12 / 22}=\left(1-P_{0}\left(\tau_{\mathrm{p}} ; \psi\right)\right) \frac{A_{3} \Omega_{3}^{2}}{A_{3}^{2}+2 \Omega_{3}^{2}} \int_{0}^{\tau_{\mathrm{p}}} d \tau \rho^{0}(\tau ; 1)_{12 / 22} .
$$

After the end of the probe pulse, any population of level 3 will rapidly decay to level 1 in a transient time of order $A_{3}^{-1}$. We denote the resulting - normalized - density matrix of the subensemble of atoms with emissions by $\tilde{\rho}$ [45]. The normalization factor of $\rho^{>}$is $1-P_{0}$, and according to Eq. (56) we obtain from Eq. (57)

$$
\tilde{\rho}\left(\tau_{\mathrm{p}} ; \psi\right)_{12 / 22}=\frac{A_{3} \Omega_{3}^{2}}{A_{3}^{2}+2 \Omega_{3}^{2}} \int_{0}^{\tau_{\mathrm{p}}} d \tau \rho^{0}(\tau ; 1)_{12 / 22} .
$$

For this we note that, by Eq. (53),

$$
\begin{aligned}
& \rho_{12}^{0}(\tau ; 1)=\left\langle 1\left|e^{-M \tau}\right| 1\right\rangle\left\langle 1\left|e^{-M \tau}\right| 2\right\rangle \\
& \rho_{22}^{0}(\tau ; 1)=\left|\left\langle 2\left|e^{-M \tau}\right| 1\right\rangle\right|^{2} .
\end{aligned}
$$


Using Eq. (44) for $e^{-M \tau}$ an elementary calculation yields

$$
\begin{aligned}
& \tilde{\rho}_{22}=\pi \frac{\tau_{\mathrm{p}}}{T_{\pi}} \epsilon_{\mathrm{p}} \quad+\text { higher orders in } \epsilon_{\mathrm{p}}, \epsilon_{\mathrm{d}} \\
& \tilde{\rho}_{12}=i \epsilon_{\mathrm{p}} \quad+\text { higher orders in } \epsilon_{\mathrm{p}}, \epsilon_{\mathrm{d}} \\
& \tilde{\rho}_{11}=1-\tilde{\rho}_{22}, \quad \tilde{\rho}_{21}=-\tilde{\rho}_{12}
\end{aligned}
$$

and its 13, 23 and 33 components vanish,

$$
\tilde{\rho}_{13}=\tilde{\rho}_{31}=\tilde{\rho}_{23}=\tilde{\rho}_{32}=\tilde{\rho}_{33}=0 .
$$

We conclude that after a short transient time at the end of the probe pulse the subensemble with photon emissions is described by the above normalized state $\tilde{\rho}$, which is independent of the initial state $|\psi\rangle$, and the relative size of the subensemble is given by $1-P_{0}\left(\tau_{\mathrm{p}} ; \psi\right)$.

We note that $\tilde{\rho}_{22}$ and $\tilde{\rho}_{12}$ are indeed very small. For the parameters of the experiment [3, 44] one has $\tilde{\rho}_{22}<1.2 \cdot 10^{-5}$ and $\left|\tilde{\rho}_{12}\right|<4.1 \cdot 10^{-4}$.

(iii) Level population after a probe pulse

We denote by $\rho^{(\mathrm{p})}$ the density matrix of the complete atomic ensemble after a short transient time at the end of a probe pulse. By the preceding results it is given by

$$
\rho^{(\mathrm{p})}=P_{0}\left(\tau_{\mathrm{p}} ; \rho^{\mathrm{in}}\right)|\tilde{\lambda}\rangle\langle\tilde{\lambda}|+\left(1-P_{0}\left(\tau_{\mathrm{p}} ; \rho^{\mathrm{in}}\right)\right) \tilde{\rho}
$$

where $\rho^{\text {in }}$ is the density matrix at the beginning of the probe pulse. From Eqs. (52), (51) and $(60)$ one immediately obtains for the population of level 2 after the probe pulse

$$
\rho_{22}^{(\mathrm{p})}=\rho_{22}^{\mathrm{in}}+2 \epsilon_{\mathrm{p}} \operatorname{Im} \rho_{12}^{\mathrm{in}}+\pi \frac{\tau_{\mathrm{p}}}{T_{\pi}} \epsilon_{\mathrm{p}}\left(1-2 \rho_{22}^{\mathrm{in}}\right) .
$$

The first term is the projection-postulate result for an ideal measurement. For $n$ probe pulses, $\rho_{12}$ is of the order $\sin \frac{\pi}{2}\left(\frac{1}{n}-\frac{\tau_{\mathrm{p}}}{T_{\pi}}\right)$, and if $n$ is as in the experiment [3] the second term is larger than, or comparable to, the last term. In the corresponding Eq. (16) of Ref. [5] this important term is missing, due the approximation used there [22], and the correction appearing there is equivalent to $O\left(\epsilon_{\mathrm{p}} 2 \pi \tau_{\mathrm{p}} / T_{\pi}\right)$ in our notation.

Summarizing this section, we have shown that, for the parameters $\epsilon_{\mathrm{p}}$ and $\epsilon_{\mathrm{d}}$ much less than 1 , a probe pulse acts as an effective state reduction also in the presence of the $\pi$ pulse. The reductions are to $\tilde{\rho}$ and $|\tilde{\lambda}\rangle$, corresponding to subensembles with and without emissions, respectively. One has $\tilde{\rho} \approx|1\rangle\langle 1|$ and $|\tilde{\lambda}\rangle \approx|2\rangle$. The corrections have been explicitly calculated in terms of the above parameters [46].

\section{Level population after $\mathbf{n}$ probe pulses}

The preceding results show that, after a probe pulse and a short transient time, the ensemble consists of two subensembles, one in the state $\tilde{\rho}$ and the other in $|\tilde{\lambda}\rangle$, corre- 
sponding to atoms with and without photon emissions. The small difference from the projection result can have a cumulative effect for the density matrix after $n$ probe pulses.

After a probe pulse, the density matrix for the complete ensemble is of the form

$$
\rho=\alpha \tilde{\rho}+\beta|\tilde{\lambda}\rangle\langle\tilde{\lambda}|
$$

with $\alpha+\beta=1$. Until the beginning of the next probe pulse at a time $T_{\pi} / n-\tau_{\mathrm{p}}$ later the time development is given by the $\pi$ pulse only, i.e. in matrix notation and in the $|1\rangle-|2\rangle$ subspace by

$$
U_{\pi}(t, 0)=\left(\begin{array}{cc}
\cos \frac{1}{2} \Omega_{2} t & -i \sin \frac{1}{2} \Omega_{2} t \\
-i \sin \frac{1}{2} \Omega_{2} t & \cos \frac{1}{2} \Omega_{2} t
\end{array}\right) .
$$

For $t=\frac{T_{\pi}}{n}-\tau_{\mathrm{p}}$ we define $U_{\pi}(t, 0) \equiv \tilde{U}_{n}$. We will now determine the density matrix after the $k$-th probe pulse. To this end we put

$$
\begin{aligned}
p & =\operatorname{tr}\left\{\mathbb{P}_{1,2} e^{-M \tau_{\mathrm{p}}} \tilde{U}_{n} \tilde{\rho} \tilde{U}_{n}^{\dagger} e^{-M^{\dagger} \tau_{\mathrm{p}}} \mathbb{P}_{1,2}\right\} \\
q & =\| \mathbb{P}_{1,2} e^{-M \tau_{\mathrm{p}}} \tilde{U}_{n}|\tilde{\lambda}\rangle \|^{2}
\end{aligned}
$$

where $\mathbb{P}_{1,2}$ is the projector onto the $1-2$ subspace. Physically, $p$ is the probability of finding no photons after the next probe pulse [40] if one had started with $\tilde{\rho}$ at the end of the preceding probe pulse. Similarly for $q$ and $|\tilde{\lambda}\rangle$.

With the abbreviation

$$
\begin{aligned}
& s_{n}=\sin \pi\left(\frac{1}{n}-\frac{\tau_{\mathrm{p}}}{T_{\pi}}\right) \\
& c_{n}=\cos \pi\left(\frac{1}{n}-\frac{\tau_{\mathrm{p}}}{T_{\pi}}\right)
\end{aligned}
$$

one finds by a straightforward calculation from Eq. (67) for $U_{\pi}$ and Eq. (44) for $e^{-M \tau_{\mathrm{p}}}$

$$
\begin{aligned}
p & =\frac{1}{2}\left(1-c_{n}\right)+s_{n} \epsilon_{\mathrm{p}}+\tilde{\rho}_{22} c_{n}-i \tilde{\rho}_{12} s_{n}-\frac{1}{2} \pi \frac{\tau_{\mathrm{p}}}{T_{\pi}}\left(1-c_{n}\right) \epsilon_{\mathrm{p}} \\
& =\frac{1}{2}\left(1-c_{n}\right)+2 s_{n} \epsilon_{\mathrm{p}}+\pi \frac{\tau_{\mathrm{p}}}{T_{\pi}} c_{n} \epsilon_{\mathrm{p}}-\frac{1}{2} \pi \frac{\tau_{\mathrm{p}}}{T_{\pi}}\left(1-c_{n}\right) \epsilon_{\mathrm{p}} \\
q & =\frac{1}{2}\left(1+c_{n}\right)-2 s_{n} \epsilon_{\mathrm{p}}-\frac{1}{2} \pi \frac{\tau_{\mathrm{p}}}{T_{\pi}}\left(1+c_{n}\right) \epsilon_{\mathrm{p}}
\end{aligned}
$$

where higher orders in $\epsilon_{\mathrm{p}}$ and $\epsilon_{\mathrm{d}}$ have been omitted.

Now suppose that after the $(k-1)$-st probe pulse the density matrix $\rho$ is given by Eq. (66) with $\alpha=\alpha(k-1)$ and $\beta=\beta(k-1)$. Then after the $k$-th probe pulse the relative size of the no-photon subensemble is given by

$$
\begin{aligned}
\beta(k) & =p \alpha(k-1)+q \beta(k-1) \\
& =p+(q-p) \beta(k-1)
\end{aligned}
$$


where $\alpha=1-\beta$ has been used. The solution of this recurrence relation is

$$
\begin{aligned}
& \beta(k)=p \frac{1-(q-p)^{k-1}}{1-(q-p)}+(q-p)^{k-1} \beta(1) \\
& \alpha(k)=1-\beta(k) .
\end{aligned}
$$

According to Eq. (66) $\beta(1)$ is the probability to find no photon during the first probe pulse [40. For the initial condition that all atoms are prepared in the ground state at the beginning of the experiment, as in the experiment of Ref. [3], $\beta(1)$ is given by

$$
\begin{aligned}
\beta(1) & =\| \mathbb{P}_{1,2} e^{-M \tau_{\mathrm{p}}} \tilde{U}_{n}|1\rangle \|^{2} \\
& =\frac{1}{2}\left(1-c_{n}\right)+s_{n} \epsilon_{\mathrm{p}}-\frac{1}{2} \pi \frac{\tau_{\mathrm{p}}}{T_{\pi}}\left(1-c_{n}\right) \epsilon_{\mathrm{p}} .
\end{aligned}
$$

At the end of the $\pi$ pulse, i.e. after the $n$-th probe pulse the density matrix is

$$
\rho\left(T_{\pi}\right)=\alpha(n) \tilde{\rho}+\beta(n)|\tilde{\lambda}\rangle\langle\tilde{\lambda}| .
$$

The populations of levels 2 is then

$$
\rho_{22}\left(T_{\pi}\right) \equiv\left\langle 2\left|\rho\left(T_{\pi}\right)\right| 2\right\rangle=\alpha(n) \tilde{\rho}_{22}+\beta(n)|\langle 2 \mid \tilde{\lambda}\rangle|^{2} .
$$

Using Eqs. (51), (60) and Eqs. (70)-(75) and omitting higher orders in $\epsilon_{\mathrm{p}}$ and $\epsilon_{\mathrm{d}}$ one obtains

$$
\begin{aligned}
& \rho_{22}\left(T_{\pi}\right)=\frac{1}{2}\left(1-c_{n}^{n}\right)+(2 n-1) s_{n} c_{n}^{n-1} \epsilon_{\mathrm{p}}+\pi n \frac{\tau_{\mathrm{p}}}{T_{\pi}} c_{n}^{n} \epsilon_{\mathrm{p}} \\
& \rho_{11}\left(T_{\pi}\right)=1-\rho_{22}\left(T_{\pi}\right) .
\end{aligned}
$$

Omitting also the $\epsilon_{\mathrm{p}}$ terms one obtains for the populations of levels 1 and 2 the approximate results 47

$$
\begin{aligned}
& \rho_{22}\left(T_{\pi}\right) \cong \frac{1}{2}\left(1-c_{n}^{n}\right)=\frac{1}{2}\left[1-\cos ^{n} \pi\left(\frac{1}{n}-\frac{\tau_{\mathrm{p}}}{T_{\pi}}\right)\right] \\
& \rho_{11}\left(T_{\pi}\right) \cong \frac{1}{2}\left(1+c_{n}^{n}\right)=\frac{1}{2}\left[1+\cos ^{n} \pi\left(\frac{1}{n}-\frac{\tau_{\mathrm{p}}}{T_{\pi}}\right)\right] .
\end{aligned}
$$

For $n$ ideal measurements with the projection postulate the result would be [3, 7]

$$
\begin{gathered}
\rho_{22}\left(T_{\pi}\right)=\frac{1}{2}\left[1-\cos ^{n} \frac{\pi}{n}\right] \\
\rho_{11}\left(T_{\pi}\right)=\frac{1}{2}\left[1+\cos ^{n} \frac{\pi}{n}\right] .
\end{gathered}
$$

This differs from the result in Eq. (77) only by the term $\tau_{\mathrm{p}} / T_{\pi}$ in the cosine, and therefore Eq. (77) can be obtained from the ideal projection result by neglecting the action of the $\pi$ pulse during a probe pulse, thus replacing the time $\Delta T=T_{\pi} / n$ between measurements 


\begin{tabular}{|c|c|c|c|c|c|}
\hline \multicolumn{6}{|c|}{ Projection Postulate } \\
\hline$n$ & $\Delta T=T_{\pi} / n$ & $\Delta T=T_{\pi} / n-\tau_{\mathrm{p}}$ & Quantum Jump & Bloch equation. & Observed \\
\hline 1 & 1.00000 & 0.99978 & 0.99978 & 0.99978 & 0.995 \\
\hline 2 & 0.50000 & 0.49957 & 0.49960 & 0.49960 & 0.500 \\
\hline 4 & 0.37500 & 0.35985 & 0.36062 & 0.36056 & 0.335 \\
\hline 8 & 0.23460 & 0.20857 & 0.20998 & 0.20993 & 0.194 \\
\hline 16 & 0.13343 & 0.10029 & 0.10215 & 0.10212 & 0.103 \\
\hline 32 & 0.07156 & 0.03642 & 0.03841 & 0.03840 & 0.013 \\
\hline 64 & 0.00371 & 0.00613 & 0.00789 & 0.00789 & -0.006 \\
\hline
\end{tabular}

Table 2: Predicted and observed population of level 2 at the end of the $\pi$ pulse for $n$ probe pulses of length $\tau_{\mathrm{p}}$.

by the effective time $\Delta T=T_{\pi} / n-\tau_{\mathrm{p}}$. This can be also understood directly quite easily as follows. Atoms which emit photons during a probe pulse flip repeatedly between levels 1 and 3 , and so the $\pi$ pulse acts less effectively them. Moreover, right after emission of a photon an atom is in the ground state and since the action of the $\pi$ pulse is of cosine form it is small for small times. Similarly, as shown in Section 4, the atoms without emissions rapidly approach the state $|\tilde{\lambda}\rangle \cong|2\rangle$ so that one again has a small action for small times. Therefore the action of the $\pi$ pulse is greatly inhibited during a probe pulse .

It is evident from Eq. (77) that this approximation yields the same result as if one had switched off the $\pi$ pulse during the probe pulse and then uses the results of Section 3 .

The corrections of Eq. (76) to the approximate values for $\rho_{i i}\left(T_{\pi}\right)$ in Eq. (77) are small for $\epsilon_{\mathrm{p}} \ll 1$. Moreover, it is straightforward to show that in the parameter range considered here the correction to $\rho_{22}\left(T_{\pi}\right)$ is positive and increases with $n$, as long as $\tau_{\mathrm{p}}$ is not too close to $T_{\pi} / n$. This is borne out in Table 2 where predicted and observed values of $\rho_{22}\left(T_{\pi}\right)$ for the parameters of the experiment of Ref. [3] are shown. The second column is based on the projection postulate for $n$ ideal measurements. The third column is based on Eq. (77) or, alternatively, on $n$ ideal measurements with ensuing switch-off of the $\pi$ pulse for $\tau_{\mathrm{p}}$ seconds. The agreement between the quantum jump result in Eq. (76) and the numerical solution of the three-level Bloch equations of Eq. (24) in column 5 is apparent. The projection postulate with $\Delta T$ modified to $\Delta T=T_{\pi} / n-\tau_{\mathrm{p}}$ also gives very good results. The experiment deals with a system where additional energy levels may make minor contributions [10] and this may explain the deviations from the experimental results in the last column of Table 2.

\section{Conclusions. Does the Zeno effect exist?}

We have investigated the so-called quantum Zeno effect for an ensemble of atomic three-level systems as that of the experiment in Ref. [3]. There has been some controversy about the interpretation of that experiment as to whether it provides an experimental proof of that effect. The Zeno effect is a theoretical prediction for the behavior of a system 
under rapidly repeated measurements with ensuing state reductions according to the projection postulate. As explained in the Introduction, the controversy has mainly focused on two points, namely (i) whether some of the "measurements" in Ref. [3] should not rather be included as "interactions" in the Hamiltonian, and (ii) whether the projection postulate is appropriate at all.

Now, a measurement on a microscopic system is indeed a complicated interaction with another system, ultimately at the macroscopic level. Not all conceivable measurements conform to the idealized case considered by von Neumann and Lüders [2] where each measurement is associated with an operator, $A$ say, such that the mean value, $\langle A\rangle$, is given by the expectation value and the mean-square deviation by the expectation of $(A-\langle A\rangle)^{2}$. As a simple example one may consider a system of photons in a cavity. The outcome of the measurement consists of two possible pointer readings, 0 and 1 say. The actual measurement is performed by a "black box" which has been so constructed that an auxiliary two-level atom in some initial state is passed through the cavity and then it is determined whether the atom is in the ground or excited state, yielding the pointer readings 0 or 1 , respectively. Considered as a measurement performed by the black box on the photon system, it is obviously not of the above kind. There is indeed an operator $A$ for the photon system, whose expectation value gives the mean, but it has eigenvalues different from 0 and 1 , and right after a measurement the photon is not in an eigenstate of $A$. As a consequence, the mean-square deviation is not of the above form. By combining part of the black box, namely the atom, with the photons to a larger system one may possibly retrieve or come close to the situation considered by von Neumann, depending on how the measurement on the atom is actually performed.

This example shows that one may have to study the measurement at hand more closely. This is what we have done with the atomic Zeno experiment by means of the quantum jump approach [26, 27], which is essentially equivalent to the Monte-Carlo wave function approach [29] and to the quantum trajectory approach [28]. The advantage of this approach is that it allows a physically intuitive as well as analytic treatment of the problem. Part of the measurement interaction - the laser pulses - has been incorporated in the Hamiltonian; this corresponds in the above example to combining the photon system and atom to a larger system and is related to shifting the Heisenberg "cut" 48.

Our analysis has shown that, to a fair accuracy and within the parameter regimes considered in this paper, a short probe pulse can indeed be viewed as performing a measurement of levels 1 or 2 of the atom, with ensuing state reduction, as given by the projection postulate for an ideal measurement. However, since these "measuring" pulses have been modeled very accurately in the Hamiltonian we were able to show that the more realistic case is also slightly more complicated, giving rise to corrections to the idealized case. These corrections were explicitly calculated, and they have a cumulative effect on the density matrix when the number of probe pulses is increased - i.e. just for the interesting case.

What then remains of the Zeno effect? Does it exist at all? In our opinion the answer depends on one's point of view. If one takes the view that for example the probe pulses ("measuring pulses") have nothing to do with a measurement but just lead to additional terms in the Hamiltonian, then any change in the temporal development is not surprising and may simply be attributed to these additional interaction terms. The other - possibly more fruitful - point of view is that these pulses approximately realize measurements with state reductions, and then one immediately has simple predictions for 
the approximate behavior of the system and arrives at the impediment and slow-down of the time evolution without complicated calculation. Finer details require of course a finer analysis, as performed in the previous sections. An actual freezing of the state does not seem possible since all realistic measurements take a finite time. In the present case this hinges on the corrections and on the finite duration of the probe pulse (including the transient decay time of order $A_{3}^{-1}$ ).

Our analysis may possibly shed some light on the use of the projection postulate in quantum optics in general, not only in connection with the Zeno effect. It seems that quite often the projection postulate is a useful tool which can give quick and fairly accurate answers. The accuracy depends on how far the particular realistic measurement differs from an ideal measurement as considered in orthodox quantum mechanics, and corrections may have to be taken into account. The idealization of realistic measurements and the projection postulate may often be very useful. Over-idealization, however, should be avoided since any idea, when carried to extremes, easily reduces itself to absurdity.

Acknowledgments. One of us (G.C.H) is indebted to the late Gerhart Lüders (1920 - 1995), who also discovered the TCP Theorem, for stimulating discussions on his formulation of the projection postulate.

\section{References}

[*] e-mail: beige@theorie.physik.uni-goettingen.de and hegerf@theorie.physik.uni-goettingen.de

[1] B. Misra and E.C.G. Sudarshan, J. Math. Phys. 18, 756 (1977)

[2] The projection postulate as commonly used nowadays is due to G. Lüders, Ann. Phys. 8, 323 (1951). For observables with degenerate eigenvalues his formulation differs from that of J. von Neumann, Mathematische Grundlagen der Quantenmechanik, Springer (Berlin 1932) (English translation:; Mathematical Foundations of Quantum Mechanics, Princeton University Press, 1955), Chapter V.1. The projection postulate intends to describe the effects of an ideal measurement on the state of a system, and it has been widely regarded as a useful tool. Lüders stressed its provisional character: "The projection postulate will be employed only until a better understanding of the actual measurement process has been found" (G. Lüders, private communication to G.C.H.). It is also known that one can envisage more general measurements where the projection postulate is not applicable; cf. e.g. G. Ludwig, Foundations of Quantum Mechanics, Vol. 1, Springer (Berlin 1983); G. Ludwig, Einführung in die Grundlagen der Theoretischen Physik, Band 3, Kap. XII, Vieweg (Braunschweig 1976); K. Kraus, States, Effects and Operations, Springer (Berlin 1983).

[3] W.M. Itano, D.J. Heinzen, J.J. Bollinger, and D.J. Wineland, Phys. Rev. A 41, 2295 (1990)

[4] V. Frerichs and A. Schenzle, Phys. Rev. A 44, 1962 (1991) 
[5] E. Block and P.R. Berman, Phys. Rev. A 44, 1466 (1991)

[6] P. Knight, Nature 344, 493 (1990)

[7] R.J. Cook, Phys. Scr. T21, 49 (1988)

[8] A $\pi$ pulse, here an rf pulse, transforms the initial state $|1\rangle$ into $|2\rangle$ at the end of the pulse, if no measurements are performed.

[9] In particular the latter part is not quite obvious for a superposition. This will be discussed in Section 3.

[10] For the influence of additional levels see Ref. [3] and L.R. Brewer, J.D. Prestage, J.J. Bollinger, W.M. Itano, and D.J. Larson, Phys. Rev. A 38, 859 (1988); R.G. Hulet, D.J. Wineland, J.C. Berquist, and W.M. Itano, Phys.Rev.A 37, 4544 (1988); D.J. Wineland, J.C. Berquist, and W.M. Itano, Optics Lett. 5, 245 (1980).

[11] A. Peres and A. Ron, Phys. Rev. A 42, 5720 (1990)

[12] L.E. Ballentine, Found. Phys. 20, 1329 (1990)

[13] L.E. Ballentine, Phys. Rev. A 43, 5165 (1991). For a response see W.M. Itano, D.J. Heinzen, J.J. Bollinger, and D.J. Wineland, Phys. Rev.A 43, 5168 (1991)

[14] D. Home and M.A.B. Whitaker, J. Phys. A 25, 657 (1992)

[15] T. Petrosky, S. Tasaki, and I. Prigogine, Phys. Lett. A 151, 109 (1990); T. Petrosky, S. Tasaki, and I. Prigogine, Physica A 170, 306 (1991)

[16] M.J. Gagen and G.J. Milburn, Phys. Rev. A 47, 1467 (1993)

[17] A.L. Rivera and S.M. Chumakov, Journ. of Mod. Optics 41, 839 (1994)

[18] E. Eberle, Lett. Nuov. C 20, 272 (1977); G.C. Ghirardi, C. Omero, T. Weber, and A. Rimini, Nuovo Cim. A 52, 421 (1979); K. Kraus, Found. Phys. 11, 547 (1981); R.A. Harris and L. Stodolsky, Phys. Lett. B 116, 464 (1982); C.B. Chiu, B. Misra, and E.C.G. Sudarshan, Phys. Lett. B 117, 34 (1982); A. Sudbery, Ann. Phys. 157, 512 (1984); E. Joos, Phys. Rev. D 29, 1626 (1984); D.P.L. Castrigiano and U. Mutze, Phys. Rev. A 30, 2210 (1984); W.C. Schieve, L.P. Horwitz, and J. Levitan, Phys. Lett. A 136, 264 (1989); R.H. Dicke, Found. Phys. 19, 385 (1989); M. Damnjanovic, Phys. Lett. A 149, 333 (1990); T.F. Jordan, E.C.G. Sudarshan, and P. Valanju, Phys. Rev. A 44, 3340 (1991); D.I. Fivel, Phys. Rev. Lett. 67, 285 (1991); G. Groessing and A. Zeilinger, Physica D 50, 321 (1991); H. Fearn and W.E. Lamb, Phys. Rev. A 46, 1199 (1992); M.J. Gagen, H.M. Wiseman, and G.J. Milburn, Phys. Rev. A 48, 132 (1993); S. Pascazio, M. Namiki, G. Badurek, and H. Rauch, Phys. Lett. A 179, 155 (1993); R. Onofrio, C. Presilla, and U. Tambini, Phys. Lett. A 183, 135 (1993); P. Blanchard and A. Jadczyk, Phys. Lett. A 183, 272 (1993); G.S. Agarwal and S.P. Tewari, Phys. Lett. A 185, 139 (1994); T.P. Spiller, Phys. Lett. A 192, 163 (1994); T.P. Altenmüller and A. Schenzle, Phys. Rev. A 49, 2016 (1994); K. Urbanowski, Phys. Rev. A 50, 2847 (1994); J.I. Cirac, A. Schenzle, and P. Zoller, Europhys. Lett. 27, 123 (1994); L.S. Schulman, A. Rafagni, and D. Mugnai, Phys. Scr. 49, 536 (1994) 
[19] After completion of this paper we received a preprint on the QZE by W.L. Power and P.L. Knight with numerical simulations of quantum trajectories.

[20] Another effect intimately related to the Zeno effect is the so-called inverse quantum Zeno effect proposed by V. Aharonov and M. Vardi, Phys. Rev. D 21, 2235 (1980) according to which a system can be made to follow an arbitrarily chosen trajectory by performing a suitable sequence of measurements. T.P. Altenmüller and A. Schenzle, Phys. Rev. A 48, 70 (1993) have discussed this inverse effect for a 2 level system.

[21] Ref. [16] also uses extensive numerical simulations of quantum trajectories to gain insight into the evolution of the atomic-level population when the system is continuously observed. We, on the other hand, use quantum trajectories in an analytic way.

[22] From this one can easily calculate the populations of levels 1 and 2 after a single probe pulse, and they turn out to differ from Eqs. (11) - (16) of Ref. [5] by an additional correction to the projection-postulate result (see our Eq. (65) below). This is due to the approximation used in Ref. [5] for the solution of the Bloch equations. This leads to a rate equation for $\rho_{22}$ in their Eq. (9) in which $\rho_{12}$ does not appear, and it introduces an error of the order of the missing term as pointed out by D.G. Sondermann (private communication).

[23] This is seen as follows. The second condition means that on average several photons should be produced during a probe pulse. Estimating the number of photons by the steady-state solution of the Bloch equations [32] for the $1 \leftrightarrow 3$ transition, driven by the probe pulse and with no population in level 2 , gives the condition

$$
A_{3} \frac{\Omega_{3}^{2}}{A_{3}^{2}+2 \Omega_{3}^{2}} \tau_{\mathrm{p}}>1,
$$

where $\Omega_{3}$ is the Rabi frequency of the probe pulse. This last condition now implies

$$
\tau_{\mathrm{p}}>\frac{A_{3}}{\Omega_{3}^{2}}+\frac{2}{A_{3}}
$$

and hence, by Eq. (11),

$$
T_{\pi}=\frac{\pi}{\Omega_{2}} \gg \frac{A_{3}}{\Omega_{3}^{2}}+\frac{2}{A_{3}} .
$$

This implies the first part of Eq. (3). The last equation can also be written as

$$
\frac{\Omega_{2}}{\Omega_{3}}\left(\frac{A_{3}}{\Omega_{3}}+2 \frac{\Omega_{3}}{A_{3}}\right) \ll \pi .
$$

Using the inequality $x+2 / x \geq 2 \sqrt{2}$ for $x>0$ this gives the condition on the Rabi frequencies in Eq. (3). This condition is also directly obtained by noting that level 1 has to be pumped at least once to level 3, and hence $\tau_{\mathrm{p}}>\pi / \Omega_{3}$. Eq. (自) then implies $\epsilon_{\mathrm{R}} \ll 1$. This condition also automatically ensures the obvious requirement that during a probe pulse the $\pi$ pulse should have negligible effect. 
[24] The case with a general $\Omega_{3}$ will be treated elsewhere.

[25] By an ensemble we always mean a quantum mechanical ensemble generated by (infinite) repetition of the preparation of single systems or by means of a collection ("gas") of noninteracting systems without cooperative effects.

[26] G.C. Hegerfeldt and T.S. Wilser 1992 in: Classical and Quantum Systems. Proceedings of the II. International Wigner Symposium, July 1991 (ed. by H.D. Doebner, W.Scherer, and F. Schroeck) World Scientific (Singapore 1992), p. 104

[27] G.C. Hegerfeldt, Phys. Rev. A 47, 449 (1993)

[28] H. Carmichael, An Open Systems Approach to Quantum Optics, Lecture Notes in Physics, Springer (Berlin 1993)

[29] J. Dalibard, Y. Castin, and K. Mølmer, Phys. Lett. 68, 580 (1992)

[30] The term "quantum jump" is ubiquitous in physics. E.g., Cook [7] defines it as the switching of the energy eigenvalue under frequent ideal measurements and uses it to calculate the density matrix of a two-level system for $n$ ideal measurements by the projection postulate. It is therefore better to speak of the "quantum jump approach in quantum optics".

[31] Note that this is applied here to photon measurements only, not to atomic measurements. These reductions can be avoided [26] by using the well-known Markov property which is also employed in the derivation of the Bloch equations as in C. Cohen Tannoudji, J. Dupont-Roc, and G. Grynberg, Atom-Photon Interactions, WileyInterscience (New York 1992).

[32] R. Loudon, The Quantum Theory of Light. 2nd Edition, Clarendon Press, London 1983

[33] See, e.g., the book in Ref. [31].

[34] Depending on the parameters there may be a finite probability that no photon is emitted at all. Therefore this probability density need not be normalized to 1 .

[35] G.C. Hegerfeldt and M.B. Plenio, Phys. Rev. A 45, 373 (1992)

[36] G.C. Hegerfeldt and M.B. Plenio, Phys. Rev. A 47, 2186 (1993)

[37] G.C. Hegerfeldt and M.B. Plenio, Quantum Optics 6, 15 (1994)

[38] G.C. Hegerfeldt and M.B. Plenio, Z. Phys. B 96, 533 (1995)

[39] For the general case cf. F.R. Gantmacher, Matrizentheorie, Springer (Berlin 1986).

[40] A small transient time after the switch-off of the probe pulse to allow for the decay of a possible 3-component is included..

[41] The eigenvalue equation is $p(\lambda)=\lambda^{3}-\frac{1}{2} A_{3} \lambda^{2}+\frac{1}{4}\left(\Omega_{2}^{2}+\Omega_{3}^{2}\right) \lambda-\frac{1}{8} A_{3} \Omega_{2}^{2}=0$. 
[42] For this one may use the identities $\sum \lambda_{i}=\operatorname{tr} M=\frac{1}{2} A_{3}, \lambda_{1} \lambda_{2}+\lambda_{2} \lambda_{3}+\lambda_{3} \lambda_{1}=$ $\frac{1}{4}\left(\Omega_{2}^{2}+\Omega_{3}^{2}\right)$, and $p\left(\lambda_{2}\right)=0$.

[43] With probability $\epsilon_{\mathrm{R}}^{2}$ a photon may be emitted by an atom during this transient time. Such an atom leaves the subensemble under consideration.

[44] In the experiment of Ref. [3] the decay rate of the third level is $1.2 \cdot 10^{8} / \mathrm{s}$. The Rabi frequency $\Omega_{3}$ is given by $1.9 \cdot 10^{6} / \mathrm{s}$, with a photon rate of 72 photons per probe pulse measured in steady state. $\Omega_{2}$ is given by $12.27 / \mathrm{s}, \tau_{\mathrm{p}}=2.4 \cdot 10^{-3} \mathrm{~s}, T_{\pi}=.256 \mathrm{~s}$, $\epsilon_{\mathrm{p}}=4.08 \cdot 10^{-4}$, and $\epsilon_{R}=6.46 \cdot 10^{-6}$.

[45] This also introduces an error of the order of $\Omega_{2} / A_{3} \ll \epsilon_{\mathrm{p}}$.

[46] The same corrections can also be obtained by an expansion in powers of $\Omega_{2}$ up to second order as follows. For an operator $L$ of the form $L=L_{0}+L_{1}$ one has the identity

$$
\exp L t=\exp L_{0} t+\int_{0}^{t} d \tau \exp L_{0}(t-\tau) L_{1} \exp L \tau
$$

as checked by differentiation. This can be iterated and applied to $\rho^{0}$ and to the complete density matrix. In both cases the corresponding $L_{1}$ is proportional to $\Omega_{2}$.

[47] Without stated explicitly, these approximate equations may also have been used in Ref. [3].

[48] von Neumann, Ref. [2], Chapter VI. 


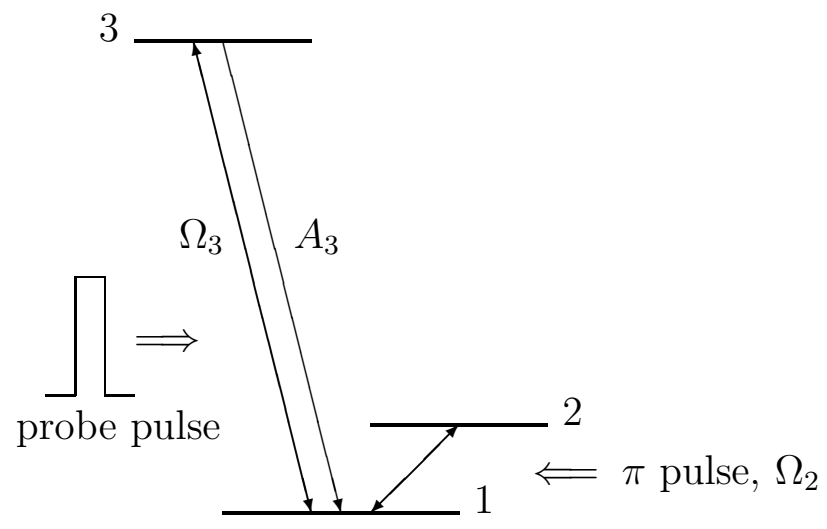

Figure 1: V system with (meta-) stable level 2 and Einstein coefficient $A_{3}$ for level $3 . \Omega_{2}$ and $\Omega_{3}$ are the Rabi frequencies of the two lasers.

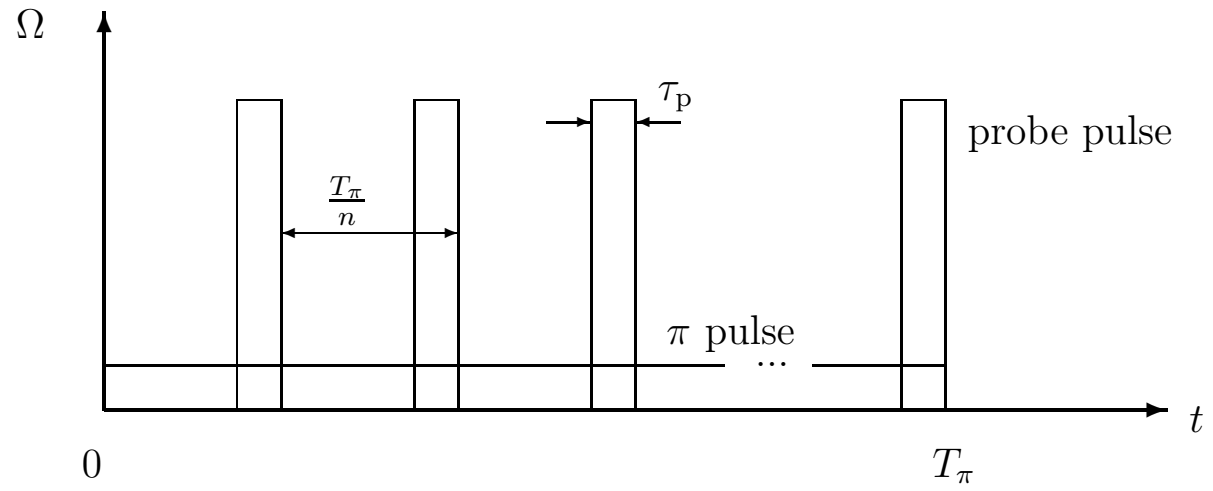

Figure 2: Probe pulses and $\pi$ pulse

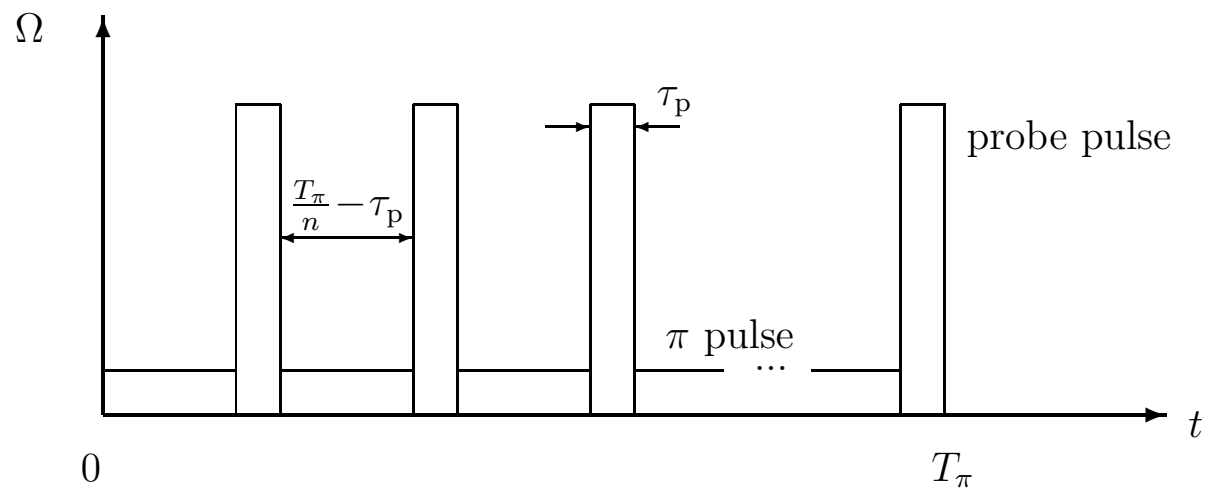

Figure 3: $\pi$ pulse switched off during probe pulse 